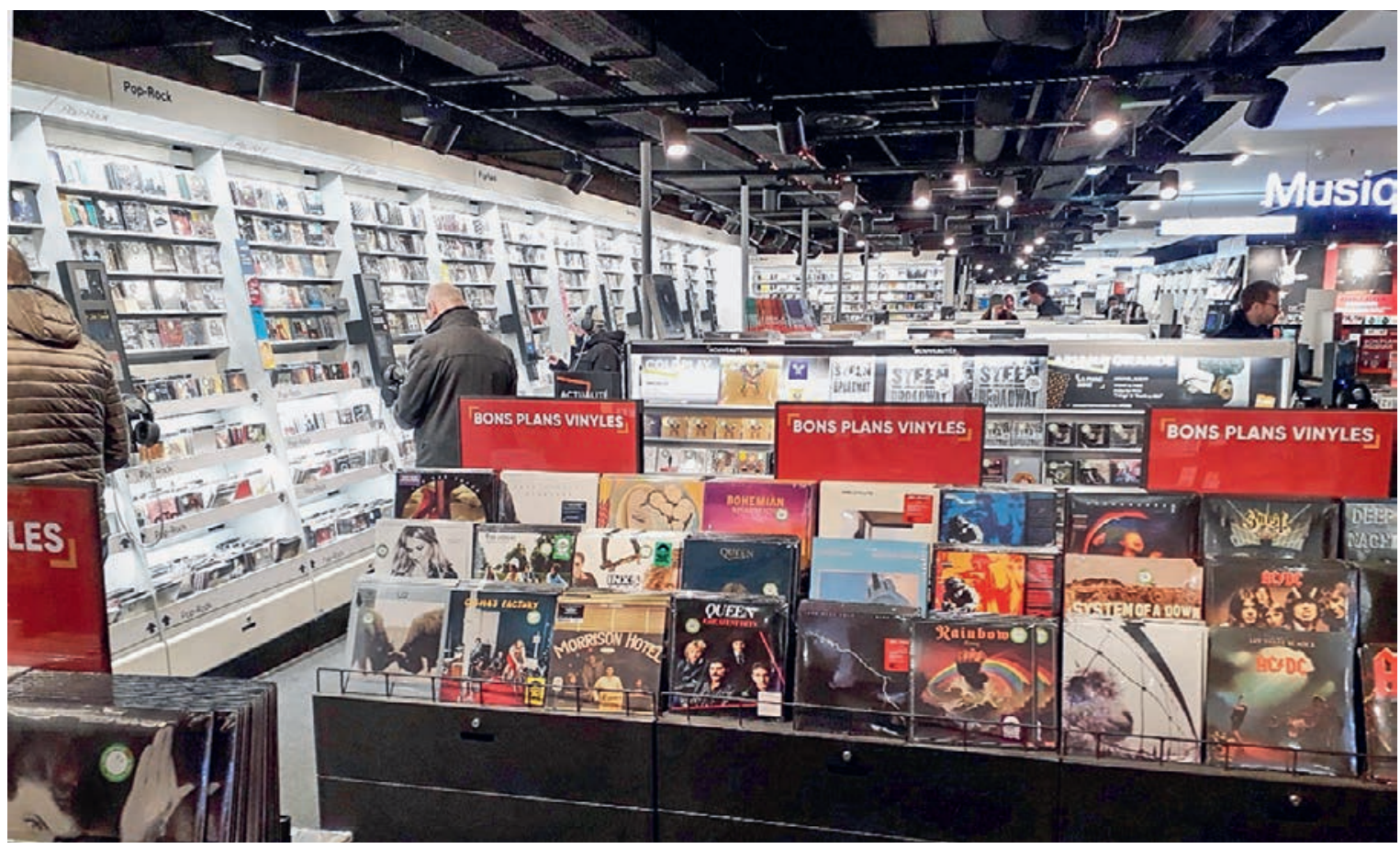

Die Vinylplatten sind zurück.

\title{
G wie Generika oder die pharmazeutische Coverversion
}

\section{Dominik Heim}

PD Dr. med., Mitglied FMH, Kompetenzzentrum für Versicherungsmedizin, Suva, Luzern

"Avant que la nuit ne s'invite, ce soir, j'ai envie d'écrire»

Johnny Hallyday, 2014

Das habe ich! Hinter uns geht die Sonne unter, im Osten ist der Himmel rabenschwarz, der TGV rast nach dem Venenkongress in Paris gegen Basel, im Ohr beschwört Johnny Hallyday das Kind, das er einmal war, «dans tout ce que tu feras, sois vrai, reste fidèle à qui tu es». Im Rucksack hat's noch weitere CD-Trouvaillen aus der Fnac (französischer Grossladen für Kultur und elektronische Gadgets): Chelsea Girl von Nico, der Muse von Velvet Underground, von 1966/1968, eine neuere Patrick Bruel, nur von Jean-Jacques Goldmann gibt es halt nichts Neues. Er hat sich 2004 aus der französischen Musikszene zurückgezogen, 1986 schrieb er Gang, das eines der bekanntesten Werke von Johnny Hallyday werden sollte. 2017 starb Johnny 74-jährig, wie ihn die Franzosen liebevoll nannten. Er war der berühmteste französische Rocker, er war kein Songwriter, das erledigten andere für ihn, er war der charismatische Franzose, der die Hits von Elvis (und vielen anderen) nach Frankreich brachte, er coverte unzählige englische Songs und wurde dafür in Frankreich verehrt. Aus dem House of the Rising Sun von den Animals wurde Le Pénitencier. Aus dem weltberühmten Original wurde eine weltberühmte, französische Coverversion ...

\section{Generika sind auch Coverversionen!}

Nach 20 Jahren ist das Patent einer Originalsubstanz frei. Dann können Generika davon produziert werden. Und der Kampf, zu diesem Zeitpunkt dann der Erste

Nach 20 Jahren ist das Patent einer Originalsubstanz frei.

auf dem Markt zu sein, ist dementsprechend gross. Sie sind rund 30\% billiger als die Originale, nicht weil die Produktion billiger ist, sondern weil eben die For- 
schung wegfällt, die haben ja andere schon gemacht. Ihre Bioverfügbarkeit beträgt $80-125 \%$ und ist somit mit den Originalen vergleichbar, erklärt Andreas Bosshard, CEO der Mepha. Dass es trotzdem noch Patienten gibt, die auf dem Original beharren, weil sie nicht an die gleiche Wirksamkeit glauben, ist deshalb nur

\section{Was ist denn eigentlich bei den Generika anders?}

schwer verständlich. Gewisse Generika, so zum Beispiel im Bereich der Asthma-Medikation, seien in der Schweiz nicht am Markt, weil Swissmedic für diese Produkte als einziges Land in Europa noch zusätzliche Studien verlange, was das Anbieten von Antiasthmatika-Generika in der Schweiz wirtschaftlich oft verunmögliche. Ja, was ist denn eigentlich bei den Generika anders? Es gibt zusätzliche Dosierungen, die Befilmung kann anders sein, die Packung ist oft besser, die Indikationsbezeichnung auf der Packung ist verständlicher - aber die Substanz ist identisch. Normalerweise ist es kein Problem, auch mitten in einer Therapie von einem Original auf ein Generikum umzusteigen, es gibt aber Ausnahmen wie zum Beispiel

Korrespondenz:

PD Dr. med. Dominik Heim heim.dominik[at]bluewin.ch bei einigen Antiepileptika, wo bei einem Wechsel die Bioverfügbarkeit sehr genau überprüft werden muss oder, noch besser, die Therapie gleich mit dem Generikum eingeleitet werde sollte. Und ja, die Gesundheitskosten kann man damit schon senken, da gibt es noch Potential. Man rechnet aktuell mit zusätzlichen Einsparungsmöglichkeiten von rund 400 Mio. CHF. Jährliche Preissenkungen von rund 5\% erhöhen dieses Einsparpotential noch weiter. Und ob diese Coverversionen denn nun besser seien? Ja, das könnten sie nun wirklich sein, gleich oder besser, schliesslich werden Generika 20 Jahre nach den Originalen entwickelt und verfügen daher in der Regel über eine modernere Technologie.

\section{Man rechnet aktuell mit zusätzlichen Einspa- rungsmöglichkeiten von rund 400 Mio. CHF.}

Da blitzt plötzlich Joe Cockers With a Little Help from My Friends vor meinem musikalischen Auge auf. Er, der sich die Seele vom Leib schreit in Woodstock, oder die dezenten, genialen Originale aus Liverpool: Original oder Generika?

PS: Wer jetzt bei der Einleitung mit dem leisen Vorwurf des ewigen Retrogefühls an den Schreiber gelangt,



